Bull. Austral. Math. Soc.

Vol. 39 (1989) [215-223]

\title{
GLOBAL DIMENSIONS OF RIGHT COHERENT RINGS WITH LEFT KRULL DIMENSION
}

\author{
MARK L. TEPLY
}

\begin{abstract}
The weak global dimension of a right coherent ring with left Krull dimension $\alpha \geqslant 1$ is found to be the supremum of the weak dimensions of the $\beta$-critical cyclic modules, where $\beta<\alpha$. If, in addition, the mapping $I \rightarrow$ assI gives a bijection between isomorphism classes on injective left $R$-modules and prime ideals of $R$, then the weak global dimension of $R$ is the supremum of the weak dimensions of the simple left $R$-modules. These results are used to compute the left homological dimension of a right coherent, left noetherian ring. Some analogues of our results are also given for rings with Gabriel dimension.
\end{abstract}

\section{INTRODUCTION}

Let $R$ be a ring with unity element and let ${ }_{R} M$ denote a left $R$-module. As usual (for example, see [5]), the weak dimension of ${ }_{R} M$ is

$$
w-\operatorname{dim} M=\inf \left\{n \geqslant 0 \mid \operatorname{Tor}_{n+1}^{R}(-, M)=0\right\}
$$

or $\infty$ if $\operatorname{Tor}_{n+1}^{R}(-, M) \neq 0$ for all $n \geqslant 0$; the projective dimension of ${ }_{R} M$ is

$$
p-\operatorname{dim} M=\inf \left\{n \geqslant 0 \mid \operatorname{Ext}_{R}^{n+1}(M,-)=0\right\}
$$

or $\infty$ if $\operatorname{Ext}_{R}^{n+1}(M,-) \neq 0$ for all $n \geqslant 0$. Then the weak and left global dimensions of $R$ are given by

$$
\text { w.gl. } \operatorname{dim} R=\sup \left\{\dot{w}-\operatorname{dim} M \mid{ }_{R} M\right\}
$$

and

$$
\text { l.gl. } \operatorname{dim} R=\sup \left\{p-\operatorname{dim} M \mid{ }_{R} M\right\} \text {. }
$$

Often 1.gl. $\operatorname{dim} R$ is computed by Auslander's classical formula as $1 . g 1 . \operatorname{dim} R=$ $\sup \left\{p-\operatorname{dim} M \mid{ }_{R} M\right.$ is cyclic $\}$. For special classes of rings the number of cyclics to be checked in computing l.gl. $\operatorname{dim} R$ may be reduced. For example if $R$ is a commutive noetherian ring, it is sufficient to check the projective dimensions of the simple modules. Let $K-\operatorname{dim} M$ denote the Krull dimension of ${ }_{R} M$ in the sense of [3], and let $1 K-$ $\operatorname{dim} R=K-\operatorname{dim}_{R} R$. Recently, Rainwater [5] proved the following related results. If $R$ is a right coherent, left noetherian ring with $1 K-\operatorname{dim} R \leqslant 1$, then 1.gl. $\operatorname{dim} R=$

Received 28 April 1988

Copyright Clearance Centre, Inc. Serial-fee code: 0004-9729/89 \$A2.00+0.00. 
$\sup \left\{p-\left.\operatorname{dim} M\right|_{R} M\right.$ simple . (Recall that $R$ is right coherent if each finitely generated right ideal is finitely presented. See [1] or [6] for some properties of right coherent rings.) If $R$ is a left fully bounded noetherian, right coherent ring, then $1 . g l . \operatorname{dim} R=$ $\sup \left\{p-\operatorname{dim} M \mid{ }_{R} M\right.$ simple $\}$.

In this paper we generalise Rainwater's result in several ways: (1) we consider w.gl. $\operatorname{dim} R$ for some nonnoetherian rings as well as l.gl. $\operatorname{dim} R$ for left noetherian rings; (2) we relax the restriction $1 K-\operatorname{dim} R \leqslant 1$; and (3) we relax the boundedness condition of Rainwater's second result in the nonnoetherian case. Some extensions to certain rings with Gabriel dimension in the sense of [3] and [4] are also observed. Thus we obtain new formulae for the weak and left global dimensions of certain right coherent rings.

We frequently use the concept of an $\alpha$-critical module; that is, a module $M$ with $K-\operatorname{dim} M=\alpha$ and $K-\operatorname{dim} N<\alpha$ for every proper homomorphic image $N$ of $M$. We use annM to denote the annihilator of a module $M$, assM to denote the assassinator of a uniform module $M$, and radR to denote the Jacobson radical of $R$.

\section{Rings with Krull dimension}

We start with two preliminary results that are used in proving our main results on the weak global dimension of rings.

LEMMA 2.1. Let $R$ be a ring with left Krull dimension, and let $M$ be a nonzero left $R$-module. Then

$w-\operatorname{dim} M \leqslant \sup \{w-\operatorname{dim} C \mid C$ is a cyclic critical subfactor of $M\}$.

Proof: Since $R$ has left Krull dimension, any nonzero left $R$-module contains a critical submodule. Hence we can form a chain of submodules of $M$ inductively as follows. Let $C_{0}=0$. For a nonlimit ordinal $\beta$, let $C_{\beta} / C_{\beta-1}$ be a critical cyclic submodule of $M / C_{\beta-1}$. For a limit ordinal $\beta$, let $C_{\beta}=\bigcup_{\gamma<\beta} C_{\gamma}$. Eventually this chain must terminate at some $C_{\delta}=M$.

Let $m=\sup \left\{w-\operatorname{dim}\left(C_{\beta} / C_{\beta-1}\right) \mid 1 \leqslant \beta \leqslant \delta, \beta\right.$ nonlimit $\}$. To avoid trivialities, we may assume that $m<\infty$ and use transfinite induction to prove that $w-\operatorname{dim} C_{\beta} \leqslant m$ for all $\beta \leqslant \delta$. By hypothesis $\operatorname{Tor}_{m+1}^{R}\left(A, C_{\beta} / C_{\beta-1}\right)=0$ for all right $R$-modules $A$; hence our induction step for nonlimit ordinals follows from the exact sequence

$$
\operatorname{Tor}_{m+1}^{R}\left(A, C_{\beta-1}\right) \rightarrow \operatorname{Tor}_{m+1}^{R}\left(A, C_{\beta}\right) \rightarrow \operatorname{Tor}_{m+1}^{R}\left(A, C_{\beta} / C_{\beta-1}\right)
$$

The induction step for limit ordinals follows immediately from the fact that $\operatorname{Tor}_{m+1}^{R}(A,-)$ commutes with direct limits. 
LEMMA 2.2. Let $R$ be a right coherent ring with left Krull dimension. Let $\alpha \geqslant 1$ and let $M$ be an $\alpha$-critical left $R$-module with prime annihilator $P$. If every singular cyclic $R / P$-module has Krull dimension $<\alpha$, then

$w-\operatorname{dim} C \leqslant \sup \{w-\operatorname{dim} C \mid C$ is a $\beta$-critical cyclic left $R$-module, $\beta<\alpha\}$.

Proof: Let $p=\sup \{w-\operatorname{dim} C \mid C$ is a $\beta$-critical cyclic left $R$-module, $\beta<\alpha\}$. Let $\left\{M_{i}\right\}_{i \in I}$ be the set of nonzero submodules of $M$. Since $\alpha \geqslant 1, M$ has no simple submodules; hence $M$ embeds in $\prod_{i \in I}\left(M / M_{i}\right)$. Thus we can find a module $L$ such that

$$
j: M \oplus L \rightarrow \prod_{i \in I}\left(M / M_{i}\right)
$$

is an essential monomorphism. Since coker $j$ is a singular $R / P$-module, it follows from our hypothesis that every critical cyclic subfactor $C$ of coker $j$ has $K-\operatorname{dim} C<\alpha$. Thus by Lemma $2.1, w-\operatorname{dim}(\operatorname{coker} j) \leqslant \sup \{w-\operatorname{dim} C \mid C$ is a cyclic critical subfactor of coker $j\} \leqslant p$. Since $K-\operatorname{dim}\left(M / M_{i}\right)<\alpha$ for each $i$, then by Lemma $2.1 w-$ $\operatorname{dim}\left(M / M_{i}\right) \leqslant p$ for each $i$. For each right $R$-module $A$, write $A=\stackrel{\lim }{\longrightarrow} A_{k}$, where each $A_{k}$ is finitely presented. Since $R$ is right coherent, then by [5, Lemma 1$]$

$$
\begin{aligned}
\operatorname{Tor}_{p+1}^{R}\left(A, \prod_{i \in I}\left(M / M_{i}\right)\right) & \cong \operatorname{Tor}_{p+1}^{R}\left(\stackrel{\lim }{\longrightarrow} A_{k}, \prod_{i \in I}\left(M / M_{i}\right)\right) \\
& \cong \stackrel{\lim }{\longrightarrow} \operatorname{Tor}_{p+1}^{R}\left(A_{k}, \prod_{i \in I}\left(M / M_{i}\right)\right) \\
& \cong \stackrel{\lim }{\longrightarrow}\left(\prod_{i \in I} \operatorname{Tor}_{p+1}^{R}\left(A_{k}, M / M_{i}\right)\right)=0
\end{aligned}
$$

Hence $w-\operatorname{dim}\left(\prod_{i \in I}\left(M / M_{i}\right)\right) \leqslant p$. From applying the long exact sequence for Tor to

$$
0 \longrightarrow M \oplus L \stackrel{j}{\longrightarrow} \prod_{i \in I}\left(M / M_{i}\right) \longrightarrow \operatorname{coker} j \longrightarrow 0
$$

it now follows that $w-\operatorname{dim} M \leqslant p$ as desired.

We can now give our first theorem on right coherent rings with left Krull dimension.

TheOREM 2.3. Let $R$ be a right coherent ring such that $I K-\operatorname{dim} R=\alpha \geqslant 1$. Then w.gl. $\operatorname{dim} R=\sup \{w-\operatorname{dim} C \mid C$ is a $\beta$-critical cyclic left $R$-module, $\beta<\alpha\}$.

Proof: Let $p=\sup \{w-\operatorname{dim} C \mid C$ is a $\beta$-critical cyclic module, $\beta<\alpha\}$. Clearly w.gl. $\operatorname{dim} R \geqslant p$. Consequently, by Lemma 2.1 , it is sufficient to assume that $p<\infty$ and to show that $w-\operatorname{dim} M \leqslant p$ for each (cyclic) $\alpha$-critical module $M$. 
Let $M$ be an $\alpha$-critical module. By [3, Theorem 8.3$]$ ass $M=P$ is a prime ideal and we may choose a nonzero cyclic submodule $N$ of $M$ such that annN $=P$. Since $K-\operatorname{dim}(M / N)<\alpha$ and $N$ is $\alpha$-critical [3, Proposition 2.3], it follows from Lemma 2.1 that there is no loss of generality in also assuming that annM is a prime ideal $P$. By [3, Proposition 6.1], every singular cyclic left $R / P$-module $C$ has $K-\operatorname{dim} C<\alpha$. Hence Lemma 2.2 yields $w-\operatorname{dim} M \leqslant p$ as desired.

Specialising Theorem 2.3 to the case $\alpha=1$ and using Lemma 2.1 for $1 K-\operatorname{dim} R=$ 0 , we obtain the following result.

CoRollary 2.4. Let $R$ be a right coherent ring with $1 . K-\operatorname{dim} R \leqslant 1$. Then

$$
w \cdot g l \cdot \operatorname{dim} R=\sup \left\{w-\operatorname{dim} S \mid{ }_{R} S \text { simple }\right\}
$$

A left noetherian ring $R$ always has left Krull dimension [3, Proposition 1.3] and w.gl. $\operatorname{dim} R=1 . g l . \operatorname{dim} R$ [6, Theorem 9.22]. Hence we also obtain the following result from Theorem 2.3.

CoROllaRY 2.5. Let $R$ be a left noetherian right coherent ring with $1 . K-\operatorname{dim} R=$ $\alpha \geqslant 1$. Then

$$
\text { l.gl. } \operatorname{dim} R=\sup \left\{p-\operatorname{dim} C \mid{ }_{R} C \text { is a } \beta \text {-critical cyclic, } \beta<\alpha\right\}
$$

For the class of left noetherian right coherent rings with $1 . K-\operatorname{dim} R \geqslant 1$, Corollary 2.5 may be viewed as an improvement of the standard result of Auslander. (See [6, Theorem 9.12].)

If we specialise Corollary 2.5 to the case $\alpha=1$ and use Lemma 2.1 for the case in which $1 . K-\operatorname{dim} R=0$, we obtain the following result of [5].

Corollary 2.6. Let $R$ be a left noetherian, right coherent ring with $1 . K-$ $\operatorname{dim} R \leqslant 1$. Then

$$
\text { l.gl. } \operatorname{dim} R=\sup \left\{p-\operatorname{dim} S \mid{ }_{R} S \text { simple }\right\} .
$$

We also note that Corollary 2.6 was known earlier [7] under the additional hypothesis that $R$ is a prime ring.

Next we consider rings whose indecomposable injective modules are uniquely associated with their assassinators. Such rings with Krull dimension are studied extensively in Chapter 8 of [3].

THEOREM 2.7. Let $R$ be a right coherent ring with left Krull dimension. Assume that the mapping $I \rightarrow$ assI gives a bijection between the isomorphism classes of indecomposable injective left $R$-modules and the prime ideals of $R$. Then

w.gl. $\operatorname{dim} R=\sup \{w-\operatorname{dim} S \mid S$ is a simple left $R$-module $\}$. 
Proof: Let $q=\sup \{w-\operatorname{dim} S \mid S$ is a simple left $R$-module $\}$. Clearly w.gl. $\operatorname{dim} R \geqslant q$. By Lemma 2.1 , it is sufficient to assume that $q<\infty$ and prove that $w-\operatorname{dim} M \leqslant q$ for every (cyclic) critical module $M$. We use induction on $K-\operatorname{dim} M$.

Since the result is trivial for 0-critical modules, we assume that $\alpha \geqslant 1, M$ is $\alpha$ critical, and $w-\operatorname{dim} C \leqslant q$ for every $\beta$-critical module $C$ with $\beta<\alpha$. By our induction hypothesis and the argument in the proof of Theorem 2.3, there is no loss of generality in assuming that annM is a prime ideal $P$. By our correspondence hypothesis and $[3$, Theoren 8.6], $K-\operatorname{dim} R / P=K-\operatorname{dim} M=\alpha$. It now follows from [3, Proposition 6.1] that every cyclic singular $R / P$-module $C$ has $K-\operatorname{dim} C<K-\operatorname{dim} R / P=\alpha$. Lemma 2.2 and our induction hypothesis now imply that

$$
w-\operatorname{dim} M \leqslant \sup \{w-\operatorname{dim} C \mid C \text { is a } \beta \text {-critical cyclic } \beta<\alpha\}=q .
$$

Since a ring with left Krull dimension whose prime factor rings are left bounded satisfies the correspondence hypothesis of Theorem 2.7, we have the following result of [5] as an immediate corollary.

CoROLlary 2.8. If $R$ is a right coherent, left fully bounded noetherian ring, then l.gl. $\operatorname{dim} R=\sup \{p-\operatorname{dim} S \mid S$ is a simple left $R$-module $\}$.

To illustrate the use of Theorem 2.7, we give the following elementary example.

Example 2.8. Let $Z$ denote the integers, let $p$ be a fixed prime, let $E=Z_{p}(\infty)$ be the indecomposable $p$-primary divisible abelian group, and let $P=Z\left[x_{1}, x_{2}, \ldots, x_{n}\right]$ be the polynomial ring in $n$ indeterminates. Then $E$ is a $P$-module via $E_{i}=0$ for each $i$. Let

$$
R=\left[\begin{array}{ll}
Z & E \\
0 & P
\end{array}\right]
$$

be a ring with the usual matrix operations. It is easy to see that $R$ is neither left nor right noetherian, but $1 . K-\operatorname{dim} R=\mathrm{r} . K-\operatorname{dim} R=n+1$. Since

$$
\left[\begin{array}{cc}
0 & 0 \\
0 & x_{i}
\end{array}\right]
$$

has non-finitely generated left annihilator

$$
A=\left[\begin{array}{ll}
Z & E \\
0 & 0
\end{array}\right]
$$

then $R$ is not left coherent. However, it is not hard to verify that each element of $R$ has finitely generated right annililator and that the intersection of any two finitely 
generated right ideals is finitely generated; thus $R$ is right coherent by [1, Theorem, 2.2]. Since $R / \mathrm{radR}$ is commutative, every prime factor ring of $R$ is bounded.

Now we compute w.gl. $\operatorname{dim} R$. First note that any simple left $R$-module $S^{\prime}$ annihilated by a (projective) left ideal of the form

$$
\left[\begin{array}{cc}
q \mathrm{Z} & E \\
0 & P
\end{array}\right]
$$

has $w-\operatorname{dim} S^{\prime} \leqslant 1$. Hence we may let $S$ be a simple left $R$-module that is annihilated by $A$. Since the left $R$-module structure and the $Z$-module structure of $A$ coincide, $\operatorname{Tor}_{1}^{R}(B, A) \neq 0$ for some $B_{R}$ and $\operatorname{Tor}_{2}^{R}(-, A)=0$. Since ${ }_{R} P \cong R / A$, we must have $\operatorname{Tor}_{3}^{R}\left(-,{ }_{R} P\right)=0$. Since $1 . g l \cdot \operatorname{dim} P=n+1$, then there is a finitely generated free resolution

$$
0 \rightarrow P_{n+1} \rightarrow P_{n} \stackrel{d_{n}}{\longrightarrow} P_{n-1} \stackrel{d_{n-1}}{\longrightarrow} \cdots \longrightarrow P_{0} \rightarrow S \rightarrow 0
$$

of $P$-modules by a Theorem of Quillen and Suslin [6, Theorem 4.63]. Letting each $P_{i}$ be a left $R$-module via $A P_{i}=0$, we get $\operatorname{Tor}_{k}^{R}\left({ }_{-}, P_{i}\right)=0$ for all $k \geqslant 3$. Using dimension shifting, we obtain $\operatorname{Tor}_{n+4}^{R}(-, S)^{-} \cong \operatorname{Tor}_{3}^{R}\left(-, P_{n+1}\right)=0$. Hence $w-\operatorname{dim} S \leqslant n+3$, and thus Theorem 2.7 implies that w.gl. $\operatorname{dim} R \leqslant n+3$.

On the other hand, if $M=P p+\sum_{i=1}^{n} P_{x_{i}}$, then there is a resolution

$$
0 \longrightarrow P_{n+1}^{\prime} \longrightarrow P_{n}^{\prime} \longrightarrow \cdots \longrightarrow P_{0}^{\prime} \stackrel{\epsilon}{\longrightarrow} P / M \longrightarrow 0
$$

of finitely generated projective $P$-modules $P_{i}^{\prime}(i=0,1, \ldots, n+1)$. Since

$$
\left[\begin{array}{ll}
0 & 0 \\
0 & P
\end{array}\right]
$$

is a direct summand of $R$ as a right $R$-module, we may also regard (*) as a projective resolution of right $R$-modules. Note that there is an inclusion $R$-homomorphism $i: P / M \rightarrow A$. If $\mu$ denotes the left multiplication map of $A$ by $\left(\begin{array}{ll}p & 0 \\ 0 & 0\end{array}\right)$, then

$$
0 \longrightarrow P_{n+1}^{\prime} \longrightarrow P_{n}^{\prime} \longrightarrow \cdots \longrightarrow P_{0}^{\prime} \stackrel{i \epsilon}{\longrightarrow} A \stackrel{\mu}{\longrightarrow} A \longrightarrow A /\left(\begin{array}{cc}
p Z & E \\
0 & 0
\end{array}\right) \longrightarrow 0
$$

is a projective resolution of right $R$-modules in which $P_{n+1}^{\prime}$ is the first flat kernel. Thus w.gl. $\operatorname{dim} R \geqslant n+3$ as desired. 


\section{Rings With Gabriel Dimension}

In this section we observe that all of our proofs in the previous section can be extended to give results about certain rings with Gabriel dimension. Two examples are given to illustrate the use of these more general results.

The properties of Gabriel dimension can be found in [3] and [4]. We use $G-\operatorname{dim} M$ to denote the Gabriel dimension of a module $M$, and $1 . G-\operatorname{dim} R=G-\operatorname{dim}_{R} R$. For a nonlimit ordinal $\alpha$, a module $M$ is called $\alpha$-simple if $G-\operatorname{dim} M=\alpha$ and every proper homomorphic image $N$ of $M$ has $G-\operatorname{dim} N<\alpha$. If $M$ is $\alpha$-simple for some $\alpha$, then $M$ is called Gabriel simple.

If we replace the $\alpha$-critical modules by $\alpha$-simple modules, then the proofs of Lemmas 2.1 and 2.2 are easily modified to give the following analogues for rings with Gabriel dimension.

LEMMA 3.1. Let $R$ be a ring with left Gabriel dimension, and let $M$ be a nonzero left $R$-module. Then

$w-\operatorname{dim} M \leqslant \sup \{w-\operatorname{dim} S \mid S$ is a cyclic Gabriel simple subfactor of $M\}$.

Lemma 3.2. Let $R$ be a right coherent ring with left Gabriel dimension. Let $\alpha \geqslant 2$, and let $M$ be an $\alpha$-simple left $R$-module with prime annihilator $P$. If every singular cyclic left $R / P$-module $C$ has $G-\operatorname{dim} C<\alpha$, then

$$
w-\operatorname{dim} M \leqslant \sup \left\{w-\left.\operatorname{dim} S\right|_{R} S \text { is a } \beta \text {-simple cyclic, } \beta<\alpha\right\} .
$$

If we use [3, Proposition 6.2] in place of [3, Proposition 6.1], it is trivial to modify the proofs of Theorems 2.3 and 2.7 to obtain the following two results.

ThEOREM 3.3. Let $R$ be a right coherent ring with left Gabriel dimension $\alpha \geqslant 2$. Assume that

(1) each $\alpha$-simple module $S$ contains a nonzero submodule $S^{\prime}$ such that ann $S^{4}=$ ass $S^{4}$, and

(2) $R /$ ass $S$ has finite uniform dimension for each $\alpha$-simple module $S$.

Then

w.gl. $\operatorname{dim} R=\sup \{w-\operatorname{dim} S \mid$ Sis a $\beta$-simple cyclic left $R$-module, $\beta<\alpha\}$.

Theonem 3.4. Let $R$ be a right coherent ring with left Gabriel dimension. Assume that

(1) each Gabriel simple $R$-module $S$ has a nonzero submodule $S^{\prime}$ such that ann $S^{\prime}=$ ass $S^{\prime}$, 
(2) R/assS has finite uniform dimension for each Gabriel simple module $S$, and

(3) $G-\operatorname{dim} S=G-\operatorname{dim}(R / a s s S)$ for each Gabriel simple $S$.

Then

w.gl. $\operatorname{dim} R=\sup \{w-\operatorname{dim} S \mid S$ is a simple left $R$-module $\}$.

To illustrate the results of this section we mention the following two examples.

Example 3.5. Let $Z$ denote the integers, let $P=Z\left[x_{1}, x_{2}, \ldots, x_{n}\right]$ be the commutative polynomial ring in $n$ indeterminates, and let $F$ be the quotient field of $P$. Let $B=\bigoplus_{i=1}^{\infty} F$, and make $B$ into a module over the polynomial ring $F[y]$ via $B y=0$. Let

$$
R=\left[\begin{array}{cc}
P & B \\
0 & F[y]
\end{array}\right]
$$

be a ring with the usual matrix operations. It is rather straightforward to verify the following statements:

(i) $1 . G-\operatorname{dim} R=\mathbf{r} \cdot G-\operatorname{dim} R=n+2$;

(ii) $R$ is right coherent, but not left coherent;

(iii) hypotheses (1) - (3) of Theorem 3.4 are satisfied, as $R / \mathrm{radR}$ is a commutative noetherian ring and $(\operatorname{radR})^{2}=0$;

(iv) by Theorem 3.4,

$$
\text { w.gl. } \operatorname{dim} R=\sup \left\{w-\operatorname{dim} S \mid{ }_{R} S \text { simple }\right\}=n+1 \text {; }
$$

(v) since the ideal

$$
\left[\begin{array}{ll}
0 & B \\
0 & 0
\end{array}\right]
$$

is a direct sum of infinitely many left and right $R$-modules, then $1 K-$ $\operatorname{dim} R$ and $\mathrm{r} K-\operatorname{dim} R$ do not exist.

Example 3.6. Let $F$ be an universal differential field with derivation $d$, and let $C=F[y, d]$ be the ring of differential polynomials. In [2] it is shown that $C$ is a simple, hereditary $V$-domain with exactly one simple left $C$-module (up to isomorphism). Let $D$ be the quotient division ring of $C$. The let

$$
R=\left[\begin{array}{ll}
C & D \\
0 & C
\end{array}\right]
$$

be the ring with the usual matrix operations. This it is rather straightforward to verify the following facts:

(1) $R$ is neither left nor right noetherian; 
(2) $1 . G-\operatorname{dim} R=$ r. $G-\operatorname{dim} R=2$;

(3) $R$ is left and right coherent;

(4) hypotheses (1) and (2) of Theorem 3.3 are satisfied, as

$$
R / \operatorname{radR} \cong C \oplus C \text { and }(\operatorname{radR})^{2}=0 ;
$$

(5) by Theorem 3.3 ,

$$
\text { w.gl. } \operatorname{dim} R=\sup \left\{w-\operatorname{dim} S \mid{ }_{R} S \text { simple }\right\}=1 \text {; }
$$

(6) if $M$ is a maximal left ideal of $C$, then

$$
\text { ass }\left(R /\left[\begin{array}{cc}
M & D \\
0 & C
\end{array}\right]\right)=\left[\begin{array}{ll}
0 & D \\
0 & C
\end{array}\right]=\operatorname{ass}\left(R /\left[\begin{array}{ll}
0 & D \\
0 & C
\end{array}\right]\right)
$$

so there are 1-simple modules and 2-simple modules with the same assassinator; thus Theorem 3.4 does not apply to $R$ in this case;

(7) $1 . K-\operatorname{dim} R$ and $\mathrm{r} . K-\operatorname{dim} R$ do not exist, for $\left[\begin{array}{ll}0 & D \\ 0 & 0\end{array}\right] /\left[\begin{array}{ll}0 & C \\ 0 & 0\end{array}\right]$ has infinite uniform dimension.

\section{REFERENCES}

[1] S.U. Chase, 'Direct products of modules', Thans. Amer. Math. Soc. 87 (1960), 457-473.

[2] J. Cozzens, 'Homological properties of the ring of differential polynomials', Bull. Amer. Math. Soc. 76 (1970), 75-79.

[3] R. Gordon and J.C. Robson, Krull dimension 133 (Mem. Amer. Math. Soc., Providence, 1973).

[4] R. Gordon and J.C. Robson, 'The Gabriel dimension of a module', J. Algebra 29 (1974), 459-473.

[5] J. Rainwater, 'Global dimension of fully bounded Noetherian rings', Comm. Algebra 15 (1987), 2143-2156.

[0] J.J. Rotman, An introduction to homological algebra (Academic Press, New York, 1979).

[7] J.T. Stafford, 'Homological properties of the enveloping algebra $U\left(\mathrm{Sl}_{2}\right)$ ', Math. Proc. Cambridge Philas. Soc. 91 (1982), 29-37.

Department of Mathematical Sciences

University of Wisconsin-Milwaukee

Milwaukee, WI 53209

United States of America 